\title{
The effectiveness of a newly designed toothbrush on dental plaque removal in children
}

\author{
Hidekazu Sasaki, Mieko Matsumura, Suguru Sakashita, \\ Masato Tsuji, Michiyo Matsumoto and Takashi Ooshima \\ Department of Pediatric Dentistry, Osaka University Graduate School of Dentistry \\ 1-8 Yamada-oka, Suita, Osaka 565-0871, JAPAN
}

\begin{abstract}
Tooth brushing is accepted as an most effective measure to removal of supragingival plaque. The purpose of this study was to examine the effectiveness of a newly designed toothbrush both in brushing-simulator in vitro and in children. The newly designed toothbrush had an oval head with 8 tufts in 3 rows and cleaned the tooth surfaces of primary molar on the plastic model of the dentition more effectively in a brushing-simulator than the control toothbrush with 17 tufts in 3 rows. A total of 66 children participated the study to examine the effectiveness of the brush. The newly designed brush could remove the plaque more effectively from the primary tooth surfaces, especially upper buccal and lower lingual surfaces, than the control brush did. These results indicate that the newly designed toothbrush can remove supragingival plaque effectively in children.
\end{abstract}

\author{
Key words \\ Children, \\ Dental plaque, \\ Primary tooth, \\ Toothbrush
}

\section{Introduction}

Oral hygiene is accepted as the most important in the prevention of oral disease. Especially, mechanical tooth cleaning remains the most reliable method of controlling supragingival plaque. Although there is little scientific evidence that toothbrushing can prevent dental caries, it is demonstrated that a relationship between non-brushing habits and early caries development in infants and toddlers ${ }^{1)}$. There is no doubt that the well motivated, properly instructed children who are willing to invest time and effort in toothbrusing, can prevent dental caries ${ }^{2)}$. The universally accepted instrument for mechanical removal of dental plaque is the toothbrush. The parts of a toothbrush include the bristle head, the shank, and the handle. Variations in manual brushes may occur both in the shape and size of the handle and bristles. In the present study, significant modifications were introduced to the bristle of manual toothbrush for

Received on July 28, 2003

Accepted on November 14, 2003 children. The purpose of the present study was to examine the effectiveness of this newly designed toothbrush in the dental plaque removal in children.

\section{Materials and methods}

\section{Toothbrushes}

A toothbrush was newly designed to improve its brushing efficiency. The brush has an over-all length of $135 \mathrm{~mm}$ and an oval head $(6.5 \times 12.2 \mathrm{~mm})$ with 8 tufts in 3 rows with serrated trim of end-rounded nylon bristles (Fig. 1a). A tuft was composed of 94 bristles and planted in the rectangular holes (Fig. 1b). The diameter of each bristle was $0.178 \mathrm{~mm}$, and the maximum trim height $7.0 \mathrm{~mm}$. A conventional manual toothbrush was used as a control. The control toothbrush has an over-all length of $139 \mathrm{~mm}$ and a long oval head $(6.5 \times 14.5 \mathrm{~mm})$ with 17 tufts in 3 rows with straight trim of end-rounded nylon bristles (Fig. 1c). A tuft was composed of 60 bristles and planted in the round holes (Fig. 1d). The diameter of each bristle was $0.178 \mathrm{~mm}$ and the trim height $7.0 \mathrm{~mm}$. 

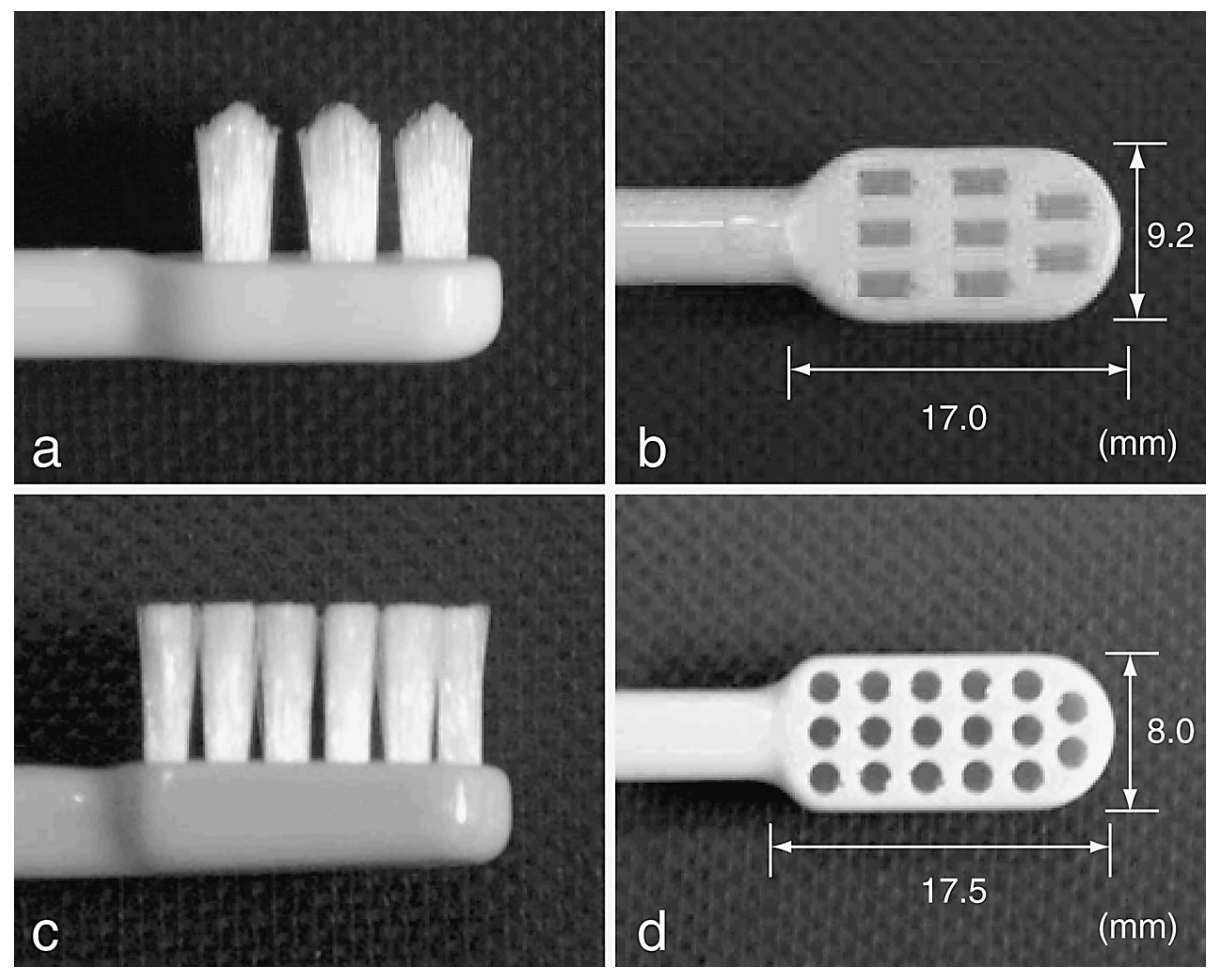

Fig. 1 Head and side views of each type of toothbrush (a, b) newly designed, (c, d) control

\section{Examination by brushing-simulator in vitro}

The effectiveness of each toothbrush was examined by brushing-simulator ${ }^{3)}$. The simulator moved the toothbrush horizontally by $10 \mathrm{~mm}$ with a load of $250 \mathrm{~g}$ at $120 \mathrm{rpm}$. The lower second primary molar on the plastic model of the dentition was stained with Occlude $^{\circledR}$ (Pascal Co., Bellevue, WA, USA). Afterwards, the stains were removed by the toothbrush in the simulator for 6 second. The areas of the occlusal and buccal surfaces where the stains remained were calculated by picture analysis. Brushing effectiveness was calculated by dividing the area of the tooth surface cleaned by the toothbrush with the area of the tooth surface stained before brushing, and expressed as mean percentage \pm standard deviation.

\section{Subjects}

Sixty six children (36 male and 30 female) of the ages between 5 and 6 years were selected from volunteers recruited from the private kindergarten in Osaka. To be selected, subjects with fully restored resin and stainless steel crowns were excluded. Ethical approval for the study was obtained from the
Ethical Committee of Osaka University Graduate School of Dentistry. The procedures, possible discomforts or risks, as well as possible benefits were fully explained to the their parents according to the guidelines established by the Japanese Public Health Service and the Osaka University Health Guidelines for Studies Involving Human Subjects. The parents gave signed and witnessed agreement to participate after receiving written information concerning the study.

\section{Study protocol}

The children received a standardized instruction for the oral hygiene method by their own toothbrush. Afterwards, they had a newly designed toothbrush and instructed to use it at home for a week. After one week use at home, each subject received an oral examination, and plaque score was calculated by the modified method of Quigley and Hein ${ }^{4}$. Plaque on the buccal, occlusal and lingual surfaces of natural teeth was stained with plaque disclosing agent (Red-Cote ${ }^{\circledR}$, John O. Butler Co., Chicago, Ill, USA) and classified into 6 grades (grade $0-5$ ) as follows; 0 : no stains, 1 : flecks of stains at gingival margin or 
Table 1 Effectiveness of newly designed toothbrush in the brushing-simulator

\begin{tabular}{lccc}
\hline Toothbrush & $\begin{array}{c}\text { Pre-Brushing } \\
\text { (pixel) }\end{array}$ & $\begin{array}{c}\text { Post-Brushing } \\
\text { (pixel) }\end{array}$ & Percent removal \\
\hline $\begin{array}{l}\text { Buccal surface } \\
\text { Newly designed }\end{array}$ & $99982 \pm 941$ & $7100 \pm 901$ & $92.9 \pm 1.0^{* * * *}$ \\
Control & $94616 \pm 3211$ & $13248 \pm 874$ & $86.0 \pm 0.9$ \\
Occlusal surface & & & \\
Newly designed & $120120 \pm 857$ & $14731 \pm 4423$ & $87.7 \pm 3.7^{* *}$ \\
Control & $126373 \pm 1722$ & $37322 \pm 5414$ & $70.5 \pm 4.3$ \\
\hline
\end{tabular}

There were statistically significant differences in percent removals between newly designed and control brushes. (**: $P<0.01$, ***: $P<0.001 ; t$-test)

Table 2 Effectiveness of newly designed toothbrush in children

\begin{tabular}{|c|c|c|c|c|c|c|}
\hline \multirow{2}{*}{$\begin{array}{l}\text { Tooth } \\
\text { Surface }\end{array}$} & \multicolumn{3}{|c|}{ Control toothbrush } & \multicolumn{3}{|c|}{ Newly designed toothbrush } \\
\hline & $\begin{array}{c}\text { Pre-Brushing } \\
\text { Score }\end{array}$ & $\begin{array}{c}\text { Post-Brushing } \\
\text { Score }\end{array}$ & $\begin{array}{l}\text { Reduction } \\
\text { Score }\end{array}$ & $\begin{array}{c}\text { Pre-Brushing } \\
\text { Score }\end{array}$ & $\begin{array}{c}\text { Post-Brushing } \\
\text { Score }\end{array}$ & $\begin{array}{l}\text { Reduction } \\
\text { Score }\end{array}$ \\
\hline \multicolumn{7}{|l|}{ Upper } \\
\hline Buccal & $2.91 \pm 0.94$ & $1.57 \pm 1.00$ & $1.41 \pm 0.71$ & $2.89 \pm 0.85$ & $0.98 \pm 0.76^{* * *}$ & $1.91 \pm 0.73 * * * *$ \\
\hline Occlusal & $2.09 \pm 0.80$ & $1.08 \pm 0.64$ & $1.02 \pm 0.65$ & $2.26 \pm 0.73$ & $0.99 \pm 0.63$ & $1.27 \pm 0.62 * *$ \\
\hline Palatal & $1.76 \pm 0.52$ & $0.91 \pm 0.63$ & $0.85 \pm 0.41$ & $1.93 \pm 0.59$ & $0.83 \pm 0.68$ & $1.10 \pm 0.52 * *$ \\
\hline \multicolumn{7}{|l|}{ Lower } \\
\hline Buccal & $2.24 \pm 0.79$ & $1.20 \pm 0.70$ & $1.05 \pm 0.61$ & $2.52 \pm 0.86$ & $1.14 \pm 0.71$ & $1.37 \pm 0.55^{* * *}$ \\
\hline Occlusal & $1.94 \pm 0.79$ & $0.84 \pm 0.48$ & $1.10 \pm 0.69$ & $2.14 \pm 0.81$ & $0.63 \pm 0.53 * *$ & $1.51 \pm 0.76^{* *}$ \\
\hline Lingual & $2.24 \pm 0.63$ & $0.87 \pm 0.77$ & $1.37 \pm 0.69$ & $2.24 \pm 0.57$ & $0.49 \pm 0.49 * * * *$ & $1.75 \pm 0.52 * * *$ \\
\hline Total & $2.25 \pm 0.54$ & $1.11 \pm 0.59$ & $1.15 \pm 0.41$ & $2.36 \pm 0.60$ & $0.85 \pm 0.52 * *$ & $1.51 \pm 0.39 * * * *$ \\
\hline
\end{tabular}

Statistical analyses were carried out between the control and new designed brushes in pre-brushing, post-brushing, and reduction score. (**: $P<0.01$, ***: $P<0.001$, ****: $P<0.0001$; Wilcoxon test)

fissures, 2: definite line of stains at gingival margin or fissures, 3: stains at less than one third of tooth surface, 4: stains at less than two third of tooth surface, 5: stains at greater than two third of the tooth surface. After scoring of pre-brushing plaque, the children were asked to brush their teeth for 5 min by themselves without looking at the stains in a mirror and using tooth paste. After brushing by themselves, post-brushing plaque scores were again calculated. Then, the plaque deposition on the tooth surfaces of all subjects were professionally removed by dentists as completely as possible with brushing. After these procedures, the children were given a control toothbrush, and instructed to use it at home for a week. One week later, same procedures were performed, and pre- and post-brushing plaque scores were examined in each subject.

\section{Statistics}

The $t$-test and Wilcoxon test were carried out to statistically examine the differences in plaque scores in in vitro brushing-simulator and in human study, respectively.

\section{Results}

\section{Brushing-simulator study}

The results in the brushing-simulator are shown in Table 1 . The percent removal of stains by the newly designed brush was $92.9 \pm 1.0 \%$ in buccal surface, and $87.7 \pm 3.7 \%$ in occlusal surface. In the control brush, on the other hand, the percent removal was 
$86.0 \pm 0.9 \%$ in buccal surface, and was $70.5 \pm$ $4.3 \%$ in occlusal surface (Table 1). The significant differences were found between newly designed and control toothbrushes in buccal surface $(P<0.001)$ and in occlusal surface $(P<0.01)$.

\section{Clinical study}

The results in children are presented in Table 2. There were no significant differences in pre-brushing scores between the control and newly designed brushes. Post-brushing scores were lower in all the tooth surfaces with newly designed brush than those with control brush, and significant differences were found in upper buccal $(P<0.001)$, lower occlusal $(P<0.01)$, lower lingual $(P<0.0001)$, and total $(P<0.01)$. Furthermore, all the reduction scores, i.e., pre-brushing score minus post-brushing score, were significantly lower in newly designed brush than those in control brush.

\section{Discussion}

Many factors such as brushing frequency, duration, force, and technique can influence the ability of an individual to remove supragingival plaque with a manual toothbrush. The design of the toothbrush can also contribute some effectiveness in plaque removal. However, few clinical reports have objectively measured the effectiveness of one particular design of toothbrush. More than half century ago, Bass described the optimum characteristics of toothbrushes for young children ${ }^{5}$. According to his description, the brush for children has plain straighthandle, over-all length about $127 \mathrm{~mm}, 3$ rows of bristles, 6 tufts to the row, evenly spaced. High quality nylon bristles were finished to round and $8.7 \mathrm{~mm}$ length. About 80 bristles with $0.127 \mathrm{~mm}$ diameter were planted in a tuft and formed straight trim. The conventional toothbrush that roughly accord to these characteristics was used as a control. In the present study, however, newly designed brush showed more effective in removing supragingival plaque on the primary teeth of children than the control toothbrush. This may be caused by the distribution and figure of the hole for tufts in the brush head. McClure compared the tooth brushing technics for the preschool children aged between 3 and 5 years and indicated that some 3 to 5 -year-old children were completely unable to wield the toothbrush, and the horizontal scrubbing technique was the most effective for the primary dentition ${ }^{6}$. The new toothbrush was designed to distribute not only the holes for tufts but also the tufts on a parallel with the long axis of the toothbrush, since the children in the primary dentition tended to move the toothbrush horizontally. As shown in Fig. 1b, rectangular holes were distributed on a parallel with the long axis. Serrated trim was given to the bristles to clean the interproximal tooth surfaces and the fissures on the occlusal tooth surfaces more effectively, as the brushing force was not so strong as that in adults. These variations in brush head must bring higher effectiveness in tooth brushing.

\section{Acknowledgments}

This work was supported by the Fund for Scientific Promotion of Sunstar Inc. We thank Mr. Atsushi Yamamoto, Mr. Tomohisa Sugimoto and Dr. Hitoshi Matsumoto for their appropriate suggestions and manufacturing newly designed toothbrush.

\section{References}

1) Wendt, L.K., Hallonsten, A.L. and Koch, G.: Oral hygiene in relation to caries development and immigrant status in infants and toddlers. Scand $J$ Dent Res 102: 269-273, 1994.

2) Koch, G., Poulsen, S. and Twetman, S.: Caries prevention in child dental care. In: Pediatric dentistry. Munksgaard, Copenhagen, 2001, pp. 119-145.

3) Suzuki, J., Tani, Y. and Kogure, T.: A study of reproducibility of brushing by a brushing simulator. J Japan Soc Periodont 40: 226-232, 1998. (in Japanese)

4) Quigley, G.A. and Hein, J.W.: Comparative cleansing efficiency of manual and power brushing. J Am Dent Assoc 65: 40-43, 1962.

5) Bass, C.C.: The optimum characteristics of toothbrushes for personal oral hygiene. Dent Item Inter 70: 697-718, 1948.

6) McClure, D.B.: A comparison of toothbrushing technics for the preschool child. J Dent Child 33: 205-210, 1966. 